\title{
SPECTRA OF SOME SPECIAL BIPARTITE GRAPHS
}

\author{
A. R. FIUJ LAALI AND H. HAJ SEYYED JAVADI
}

Received 10 March, 2015

\begin{abstract}
Let $G=(P, Q)$ be a bipartite graph and $G^{\prime}$ be a graph obtained by joining each vertex of $P$ and $Q$ with $m$ and $s$ new vertices respectively. We obtain the characteristic, Laplacian and signless Laplacian polynomial of $G^{\prime}$. As an application, we give a simple proof for Csikvari's lemma on eigenvalues of graphs.
\end{abstract}

2010 Mathematics Subject Classification: 05C50

Keywords: polynomial, bipartite, spectra

\section{INTRODUCTION}

Throughout the paper $0_{n \times n}$ and $I_{n}$ are used respectively, for square zero and identity matrix of order $n, 0_{n}$ and $j_{n}$, respectively, stand for length-n column vectors consisting entirely of 0's and 1's. The Kronecker product $A \otimes B$ of two matrices $A\left(a_{i j}\right)$ and $B\left(b_{i j}\right)$ of order $m \times n$ and $p \times q$, respectively, is the $m p \times n q$ matrix obtained from $A$ by replacing each element $a_{i j}$ by $a_{i j} B$. This operation has the properties $(A \otimes B)^{T}=A^{T} \otimes B^{T}$ and $(A \otimes B)(C \otimes D)=(A C \otimes B D)$ whenever the products $A C$ and $B D$ exist. The latter implies $(A \otimes B)^{-1}=A^{-1} \otimes B^{-1}$ for nonsingular matrices $A$ and $B$. Moreover, if $A$ and $B$ are $n \times n$ and $p \times p$ matrices, then $\operatorname{det}(A \otimes B)=(\operatorname{det} A)^{p} .(\operatorname{det} B)^{n} . \bar{K}_{n}, K_{n}$ and $K_{n, m}$ stand for the graph with $n$ isolated vertices, complete graph and complete bipartite graph, respectively. Also we use these two notations

$$
T_{w}=\left[\begin{array}{cc}
I_{w} & 0 \\
0 & 0_{(n-w) \times(n-w)}
\end{array}\right] \quad S_{w}=\left[\begin{array}{cc}
0_{(n-w) \times(n-w)} & 0 \\
0 & I_{w}
\end{array}\right]
$$

where $T_{n}=S_{n}=I_{n}$.

Let $G$ be a graph with the vertex set $\left\{v_{1}, \ldots, v_{n}\right\}$. The adjacency matrix of $G$ is an $(n \times n)$ matrix $A(G)$ whose $(i, j)$-entry is 1 if $v_{i}$ is adjacent to $v_{j}$ and 0 ,otherwise. The characteristic polynomial of $G$, denoted by $f_{G}(\lambda)$, is the characteristic polynomial of $A(G)$. We will write it simply $f_{G}$ when there is no confusion. The roots of $f_{G}$ are called the eigenvalues of $G$. The notation $(a)^{[m]}$, means that multiplicitiy of the root $a$ is $m$. The Laplacian matrix of $G$ and the signless Laplacian matrix of $G$ are defined as $L(G)=\Delta(G)-A(G)$ and $Q(G)=\Delta(G)+A(G)$, respectively, 
where $\Delta(G)$ is the diagonal matrix whose entries are the degrees of $G$. We denote the Laplacian polynomial of $G$ by $f_{L(G)}(\mu)$ and the signless Laplacian polynomial of $G$ by $f_{Q(G)}(v)$. Denote the eigenvalues of $A(G), L(G)$ and $Q(G)$, respectively, by

$$
\begin{aligned}
& \lambda_{1}(G) \geq \lambda_{2}(G) \geq \cdots \geq \lambda_{n}(G) \\
& \mu_{1}(G) \leq \mu_{2}(G) \leq \cdots \leq \mu_{n}(G) \\
& v_{1}(G) \leq \nu_{2}(G) \leq \cdots \leq v_{n}(G) .
\end{aligned}
$$

Spectral graph theory is an area of intense study, see for instance [1,2,4-6]. Graph transformations are natural techniques for producing new graphs from old ones, and their spectra have received considerable attention in recent years. In this paper, we find the characteristic, Laplacian and signless Laplacian polynomial of some special bipartite graphs obtained from other bipartite graphs by a specific graph transformation. We describe it as follows. Let $G=(P, Q)$ be a bipartite graph with $n$ vertices which $|P|=p,|Q|=n-p$. We join each vertex of $P$ and $Q$ with $m$ and $s$ new vertices, respectively. We add $(n-p)(m-s)$ isolated vertices. This only results adding 0's to the various spectrums, but will simplify our computation. We denote the resulting graph by $G_{m s}$. By a proper labeling, the adjacency matrix of $G_{m s}$ is

$$
A\left(G_{m s}\right)=\left[\begin{array}{cc}
A(G) & D \\
D^{T} & 0
\end{array}\right],
$$

where $D=j_{m}^{T} \otimes I_{n}-\left(0_{s}^{T}, j_{m-s}^{T}\right) \otimes S_{n-p}$.

We obtain the characteristic, Laplacian and signless Laplacian polynomial of $G_{m s}$. Let $G=(P, Q)$ be a bipartite graph with $n$ vertices which $|P|=p,|Q|=n-p$. We join each vertex of $P$ and $Q$ with $m$ and $s$ new vertices respectively. From now on, we denote this graph by $G^{\prime}$. In Section 2 we give some preliminaries. In Section 3 we obtain the characteristic polynomial of $G_{m s}$. The results on $G_{m s}$ enable us to obtain the characteristic polynomial of $G^{\prime}$. As an application, we give a simple proof for Csikvari's lemma on eigenvalues of graphs ( [3] Lemma 2.8). Finally, we construct some graphs whose all nonzero eigenvalues are of the form $\pm \sqrt{m \pm \sqrt{m}}$ for any natural number $m$. In Section 4 we obtain Laplacian and signless Laplacian polynomials of $G_{m s}$ and $G^{\prime}$.

\section{Preliminaries}

Lemma 1 (Schur complement [1]). Let $A$ be an $n \times n$ matrix partitioned as

$$
\left(\begin{array}{ll}
A_{11} & A_{12} \\
A_{21} & A_{22}
\end{array}\right)
$$

where $A_{11}$ and $A_{22}$ are square matrices. If $A_{11}$ and $A_{22}$ are invertible, then 


$$
\begin{aligned}
\operatorname{det}\left(\begin{array}{ll}
A_{11} & A_{12} \\
A_{21} & A_{22}
\end{array}\right) & =\operatorname{det}\left(A_{22}\right) \operatorname{det}\left(A_{11}-A_{12} A_{22}{ }^{-1} A_{21}\right) \\
& =\operatorname{det}\left(A_{11}\right) \operatorname{det}\left(A_{22}-A_{21} A_{11}{ }^{-1} A_{12}\right)
\end{aligned}
$$

Notation 1. Let $G=(P, Q)$ be a bipartite graph with $n$ vertices such that $|P|=p$ , $|Q|=n-p$. Also $C$ is a matrix of order $p \times(n-p)$ such that

$$
A(G)=\left(\begin{array}{cc}
0 & C \\
C^{T} & 0
\end{array}\right)
$$

For

$$
f_{G}(\lambda)=\operatorname{det}\left(\begin{array}{cc}
\lambda I_{p} & -C \\
-C^{T} & \lambda I_{n-p}
\end{array}\right)
$$

with two methods of Schur complement we have:

$$
\begin{array}{r}
f_{G}(\lambda)=\lambda^{n-2 p} \operatorname{det}\left(\lambda^{2} I_{p}-C C^{T}\right)=\lambda^{n-2 p} g_{G}(\lambda) \\
=\lambda^{2 p-n} \operatorname{det}\left(\lambda^{2} I_{n-p}-C^{T} C\right)=\lambda^{2 p-n} q_{G}(\lambda) .
\end{array}
$$

where $g_{G}(\lambda)=\operatorname{det}\left(\lambda^{2} I_{p}-C C^{T}\right)$ and $q_{G}(\lambda)=\operatorname{det}\left(\lambda^{2} I_{n-p}-C^{T} C\right)$. It is clear that if $n=2 p$, then $f_{G}(\lambda)=g_{G}(\lambda)=q_{G}(\lambda)$.

\section{CharaCteristic POlynOMial of $G_{m s}$}

Theorem 1. Let $G=(P, Q)$ be a bipartite graph with $n$ vertices such that $|P|=p$ , $|Q|=n-p$. Then

$$
f_{G_{m s}}(\lambda)= \begin{cases}\lambda^{m n-n+2 p}\left(\lambda^{2}-s\right)^{n-2 p} g_{G}\left(\sqrt{\frac{\left(\lambda^{2}-m\right)\left(\lambda^{2}-s\right)}{\lambda^{2}}}\right) & n \geq 2 p \\ \lambda^{m n+n-2 p}\left(\lambda^{2}-m\right)^{2 p-n} q_{G}\left(\sqrt{\frac{\left(\lambda^{2}-m\right)\left(\lambda^{2}-s\right)}{\lambda^{2}}}\right) & n \leq 2 p .\end{cases}
$$


Proof. Let $D=j_{m}^{T} \otimes I_{n}-\left(0_{s}{ }^{T}, j_{m-s}{ }^{T}\right) \otimes S_{n-p}$. By using of Schur complement we have

$$
\begin{aligned}
f_{G_{m s}}(\lambda) & =\operatorname{det}\left(\begin{array}{cc}
\lambda I_{n}-A(G) & -D \\
-D^{T} & \lambda I_{n m}
\end{array}\right) \\
= & \lambda^{m n} \operatorname{det}\left(\left(\lambda I_{n}-A(G)\right)-D\left(\lambda I_{m n}\right)^{-1} D^{T}\right) \\
= & \lambda^{m n} \operatorname{det}\left(\left(\lambda I_{n}-A(G)\right)-\frac{1}{\lambda} D D^{T}\right) \\
= & \lambda^{m n-n} \operatorname{det}\left(\left(\lambda^{2} I_{n}-\lambda A(G)\right)-D D^{T}\right) .
\end{aligned}
$$

It is easy to see that $D D^{T}=m I_{n}-(m-s) S_{n-p}$. Since $G$ is bipartite there is a matrix $C$ of order $p \times(n-p)$ such that

$$
f_{G_{m s}}(\lambda)=\lambda^{m n-n} \operatorname{det}\left(\begin{array}{cc}
\left(\lambda^{2}-m\right) I_{p} & -\lambda C \\
-\lambda C^{T} & \left(\lambda^{2}-s\right) I_{n-p}
\end{array}\right) .
$$

There are two cases:

CASE 1. Let $n \geq 2 p$. In this case, let $\left(\lambda^{2}-s\right) I_{n-p}$ be invertible. Then by Schur complement, we have

$$
\begin{aligned}
f_{G_{m s}}(\lambda) & =\lambda^{m n-n}\left(\lambda^{2}-s\right)^{n-p} \operatorname{det}\left(\left(\lambda^{2}-m\right) I_{p}-\left(\lambda C\left(\left(\lambda^{2}-s\right) I_{n-p}\right)^{-1} \lambda C^{T}\right)\right) \\
& =\lambda^{m n-n}\left(\lambda^{2}-s\right)^{n-p} \operatorname{det}\left(\left(\lambda^{2}-m\right) I_{p}-\frac{\lambda^{2}}{\left(\lambda^{2}-s\right)}\left(C C^{T}\right)\right) \\
& =\lambda^{m n-n}\left(\lambda^{2}-s\right)^{n-p}\left(\frac{\lambda^{2}}{\left(\lambda^{2}-s\right)}\right)^{p} \operatorname{det}\left(\frac{\left(\lambda^{2}-m\right)\left(\lambda^{2}-s\right)}{\lambda^{2}} I_{p}-\left(C C^{T}\right)\right) .
\end{aligned}
$$

By Notation 1 we obtain

$$
f_{G_{m s}}(\lambda)=\lambda^{m n-n+2 p}\left(\lambda^{2}-s\right)^{n-2 p} g_{G}\left(\sqrt{\frac{\left(\lambda^{2}-m\right)\left(\lambda^{2}-s\right)}{\lambda^{2}}}\right)
$$

CASE 2. Let $n \leq 2 p$. In this case, let $\left(\lambda^{2}-m\right) I_{p}$ be invertible. Then 


$$
\begin{aligned}
f_{G_{m s}}(\lambda) & =\lambda^{m n-n}\left(\lambda^{2}-m\right)^{p} \operatorname{det}\left(\left(\lambda^{2}-s\right) I_{n-p}-\left(\lambda C^{T}\left(\left(\lambda^{2}-m\right) I_{p}\right)^{-1} \lambda C\right)\right) \\
& =\lambda^{m n-n}\left(\lambda^{2}-m\right)^{p} \operatorname{det}\left(\left(\lambda^{2}-s\right) I_{n-p}-\frac{\lambda^{2}}{\left(\lambda^{2}-m\right)}\left(C^{T} C\right)\right) \\
& =\lambda^{m n-n}\left(\lambda^{2}-m\right)^{p}\left(\frac{\lambda^{2}}{\left(\lambda^{2}-m\right)}\right)^{n-p} \operatorname{det}\left(\frac{\left(\lambda^{2}-s\right)\left(\lambda^{2}-m\right)}{\lambda^{2}} I_{n-p}-\left(C^{T} C\right)\right) .
\end{aligned}
$$

By Notation 1 we obtain

$$
f_{G_{m s}}(\lambda)=\lambda^{m n+n-2 p}\left(\lambda^{2}-m\right)^{2 p-n} q_{G}\left(\sqrt{\frac{\left(\lambda^{2}-s\right)\left(\lambda^{2}-m\right)}{\lambda^{2}}}\right) .
$$

Corollary 1. For graph $G^{\prime}$ we have

$$
f_{G^{\prime}}(\lambda)= \begin{cases}\lambda^{n(s-1)+p(m-s+2)}\left(\lambda^{2}-s\right)^{n-2 p} g_{G}\left(\sqrt{\frac{\left(\lambda^{2}-m\right)\left(\lambda^{2}-s\right)}{\lambda^{2}}}\right) & n \geq 2 p \\ \lambda^{n(s+1)+p(m-s-2)}\left(\lambda^{2}-m\right)^{2 p-n} q_{G}\left(\sqrt{\frac{\left(\lambda^{2}-m\right)\left(\lambda^{2}-s\right)}{\lambda^{2}}}\right) & n \leq 2 p .\end{cases}
$$

Proof. The result follows by removing $(n-p)(m-s)$ number of $\lambda$ 's from $f_{G_{m s}}(\lambda)$.

For $s=0$, we obtain a nice result which is due to Csikvari ([3] Lemma 2.8).

Corollary 2. For graph $G^{\prime}$, let $s=0$. Then

$$
f_{G^{\prime}}(\lambda)= \begin{cases}\lambda^{n+p(m-2)} g_{G}\left(\sqrt{\lambda^{2}-m}\right) & n \geq 2 p \\ \lambda^{n+p(m-2)}\left(\lambda^{2}-m\right)^{2 p-n} q_{G}\left(\sqrt{\lambda^{2}-m}\right) & n \leq 2 p .\end{cases}
$$

Corollary 3. All nonzero eigenvalues of the graph $G^{\prime}$ are of the form $\pm \sqrt{m}$, $\pm \sqrt{s}$ and $\pm \sqrt{\frac{m+s+\lambda_{i}{ }^{2} \pm \sqrt{\left(m+s+\lambda_{i}{ }^{2}\right)^{2}-4 m s}}{2}}$, where $\lambda_{i}$ for $i=1, \ldots, t$ are all positive eigenvalues of $G$.

Proof. It is clear from $\lambda^{2}-m=0, \lambda^{2}-s=0$ and $\frac{\left(\lambda^{2}-m\right)\left(\lambda^{2}-s\right)}{\lambda^{2}}=\lambda_{i}{ }^{2}$. 
Example 1. Let $G=K_{4,2}, m=3$ and $s=1$. Then $f_{G}(\lambda)=\lambda^{2}(\underbrace{\lambda^{2}\left(\lambda^{2}-8\right)}_{q_{G}(\lambda)})$ and $8=2 p>n=6$. We have

$$
\begin{aligned}
f_{G^{\prime}} & (\lambda)=\lambda^{12}\left(\lambda^{2}-3\right)^{2}\left(\frac{\left(\lambda^{2}-3\right)\left(\lambda^{2}-1\right)}{\lambda^{2}}\right)\left(\frac{\left(\lambda^{2}-3\right)\left(\lambda^{2}-1\right)}{\lambda^{2}}-8\right) \\
= & \lambda^{8}\left(\lambda^{2}-3\right)^{3}\left(\lambda^{2}-1\right)\left(\lambda^{4}-12 \lambda^{2}+3\right) .
\end{aligned}
$$

So the eigenvalues of the graph $G^{\prime}$ are

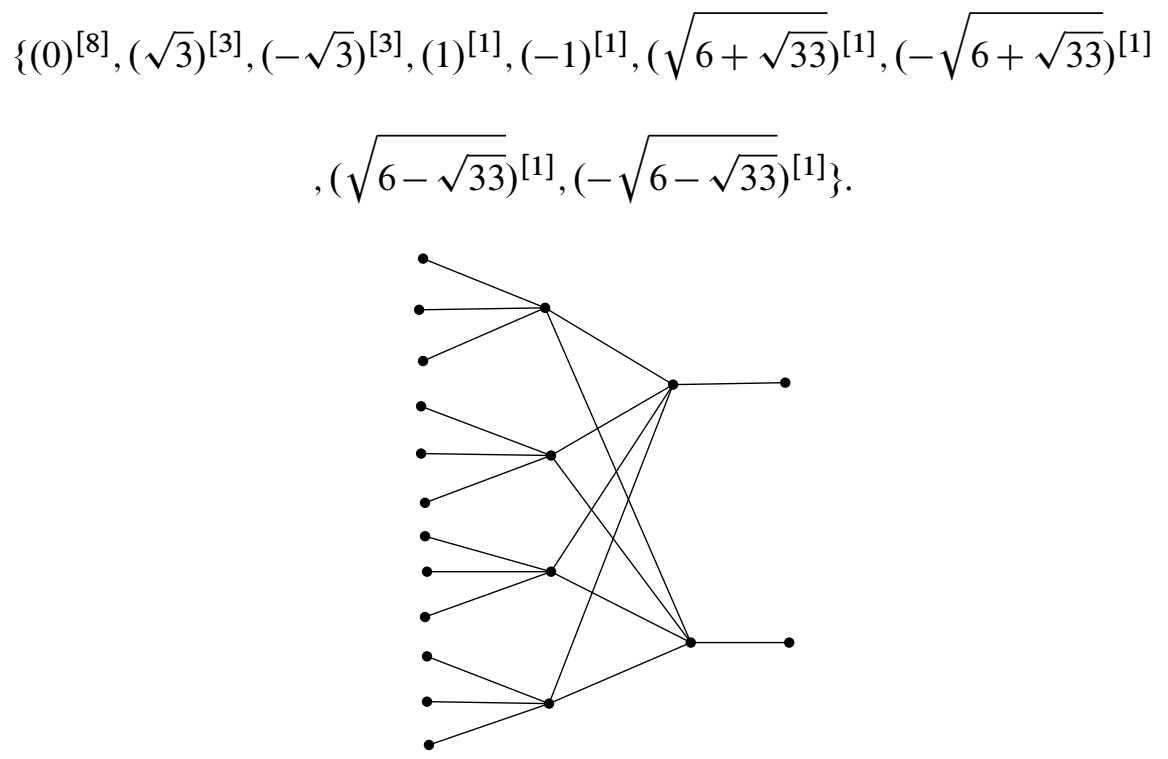

Example 2. Let $G=K_{2,4}, m=3$ and $s=1$. Then $f_{G}(\lambda)=\lambda^{2}(\underbrace{\lambda^{2}\left(\lambda^{2}-8\right)}_{g_{G}(\lambda)})$ and $4=2 p<n=6$. We have

$$
\begin{aligned}
f_{G^{\prime}}(\lambda) & =\lambda^{8}\left(\lambda^{2}-1\right)^{2}\left(\frac{\left(\lambda^{2}-3\right)\left(\lambda^{2}-1\right)}{\lambda^{2}}\right)\left(\frac{\left(\lambda^{2}-3\right)\left(\lambda^{2}-1\right)}{\lambda^{2}}-8\right) \\
= & \lambda^{4}\left(\lambda^{2}-1\right)^{3}\left(\lambda^{2}-3\right)\left(\lambda^{4}-12 \lambda^{2}+3\right) .
\end{aligned}
$$

So the eigenvalues of the graph $G^{\prime}$ are

$$
\begin{gathered}
\left\{(0)^{[4]},(1)^{[3]},(-1)^{[3]},(\sqrt{3})^{[1]},(-\sqrt{3})^{[1]},(\sqrt{6+\sqrt{33}})^{[1]},(-\sqrt{6+\sqrt{33}})^{[1]}\right. \\
\left.,(\sqrt{6-\sqrt{33}})^{[1]},(-\sqrt{6-\sqrt{33}})^{[1]}\right\} .
\end{gathered}
$$




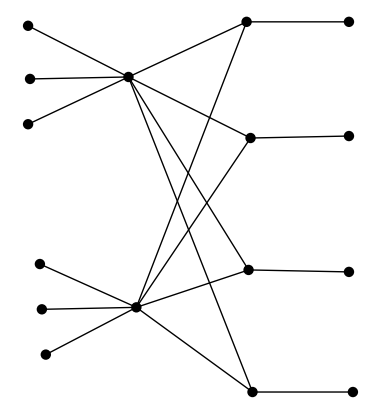

Example 3. (Graphs with eigenvalues $\pm \sqrt{m \pm \sqrt{m}}$.) For any positive integer $m$, let $G=K_{2}$ and $s=m-1$. Then by using Corollary $3, G^{\prime}$ has eigenvalue 0 with multiplicity $2 m-3$ and eigenvalues $\sqrt{m+\sqrt{m}}, \sqrt{m-\sqrt{m}},-\sqrt{m+\sqrt{m}}$ and $-\sqrt{m-\sqrt{m}}$ all with multiplicity 1 . The graph below has eigenvalue zero with multiplicity 7 , and $\sqrt{5+\sqrt{5}}, \sqrt{5-\sqrt{5}},-\sqrt{5+\sqrt{5}}$ and $-\sqrt{5-\sqrt{5}}$, all with multiplicity 1.

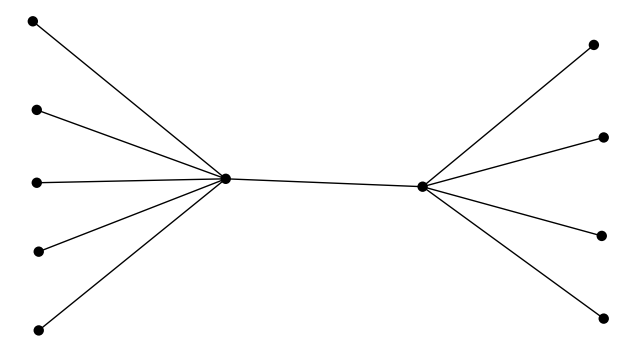

\section{LAPLACIAN AND SIGNLESS LAPLACIAN POLYNOMIAL OF $G_{m s}$}

In this section we assume that $G$ is an $r$-regular bipartite graph with $|G|=n=2 k$ where $k$ is the size of each part and $G^{\prime}$ is obtained from $G$ by joining each vertex of one part to $m$ and each vertex of another part to $s$ new vertices. We denote the Laplacian polynomial of $G^{\prime}$ by $f_{L\left(G^{\prime}\right)}(\mu)$ and the signless Laplacian polynomial of $G^{\prime}$ by $f_{Q\left(G^{\prime}\right)}(\nu)$.

Theorem 2. Let $G$ be an $r$-regular bipartite graph with $|G|=n=2 k$ where $k$ is the size of each part. Then

$$
\begin{gathered}
f_{L\left(G_{m s}\right)}(\mu) \\
=\mu^{(m-s) k}(\mu-1)^{n s+(m-s) k} f_{G}\left(\sqrt{\left(\mu-m-r-\frac{m}{\mu-1}\right)\left(\mu-s-r-\frac{s}{\mu-1}\right)}\right)
\end{gathered}
$$


Proof. We have

$$
\Delta\left(G_{m s}\right)=\left[\begin{array}{cc}
(m+r) I_{n}-(m-s) S_{k} & 0 \\
0 & B
\end{array}\right], B=\left[\begin{array}{cc}
I_{s n} & 0 \\
0 & I_{m-s} \otimes T_{k}
\end{array}\right],
$$

where $\Delta$ is diagonal matrix whose entries are the degrees of $G_{m s}$. Since $L\left(G_{m s}\right)=$ $\Delta\left(G_{m s}\right)-A\left(G_{m s}\right)$ we have

$$
\begin{aligned}
& f_{L\left(G_{m s}\right)}(\mu)=\operatorname{det}\left(\mu I-L\left(G_{m s}\right)\right)=\operatorname{det}\left(\mu I-\Delta\left(G_{m s}\right)+A\left(G_{m s}\right)\right) \\
&=\operatorname{det}\left[\begin{array}{cc}
\mu I_{n}-(m+r) I_{n}+(m-s) S_{k}+A(G) & D \\
D^{T} & \mu I_{n m}-B
\end{array}\right] .
\end{aligned}
$$

By Schur complement we have

$$
\begin{gathered}
f_{L\left(G_{m s}\right)}(\mu)=\operatorname{det}\left(\mu I_{m n}-B\right) \cdot \operatorname{det}\left(\mu I_{n}-(m+r) I_{n}+(m-s) S_{k}\right. \\
\left.+A(G)-D\left(\mu I_{m n}-B\right)^{-1} D^{T}\right)
\end{gathered}
$$

where $D=j_{m}^{T} \otimes I_{n}-\left(0_{s}^{T}, j_{m-s}^{T}\right) \otimes S_{n-p}$. It is easily seen

$$
\operatorname{det}\left(\mu I_{m n}-B\right)=\mu^{(m-s) k}(\mu-1)^{n s+(m-s) k} .
$$

Now we need to compute $D\left(\mu I_{m n}-B\right)^{-1} D^{T}$. It is easy to see that

$$
\left(\mu I_{m n}-B\right)^{-1}=\left[\begin{array}{cc}
\frac{1}{\mu-1} I_{s n} & 0 \\
0 & I_{m-s} \otimes E
\end{array}\right],
$$

where $E=\frac{1}{\mu-1} T_{k}+\frac{1}{\mu} S_{k}$. So

$$
\begin{aligned}
& D\left(\mu I_{m n}-B\right)^{-1} D^{T} \\
& =[\underbrace{I_{n}, \ldots, I_{n}}_{\text {stimes }}, \underbrace{T_{k}, \ldots, T_{k}}_{m-s t i m e s}]\left[\begin{array}{cc}
\frac{1}{\mu-1} I_{s n} & 0 \\
0 & I_{m-s} \otimes E
\end{array}\right][\underbrace{I_{n}, \ldots, I_{n}}_{\text {stimes }}, \underbrace{T_{k}, \ldots, T_{k}}_{m-s t i m e s}]^{T} \\
& =[\underbrace{\frac{1}{\mu-1} I_{n}, \ldots, \frac{1}{\mu-1} I_{n}}_{\text {stimes }}, \underbrace{\frac{1}{\mu-1} T_{k}, \ldots, \frac{1}{\mu-1} T_{k}}_{m-s t i m e s}][\underbrace{I_{n}, \ldots, I_{n}}_{\text {stimes }}, \underbrace{T_{k}, \ldots, T_{k}}_{m-s t i m e s}]^{T} \\
& =\frac{s}{\mu-1} I_{n}+\frac{m-s}{\mu-1} T_{k} .
\end{aligned}
$$

Since $G$ is bipartite, there is a matrix $C$ of order $n \times(n-p)$ such that 


$$
\begin{aligned}
& \operatorname{det}\left(\mu I_{n}-(m+r) I_{n}+(m-s) S_{k}+A(G)-D\left(\mu I_{m n}-B\right)^{-1} D^{T}\right) \\
& =\operatorname{det}\left(\mu I_{n}-(m+r) I_{n}+(m-s) S_{k}+A(G)-\left(\frac{s}{\mu-1} I_{n}+\frac{m-s}{\mu-1} T_{k}\right)\right) \\
& =\operatorname{det}\left[\begin{array}{cc}
\left(\mu-m-r-\frac{m}{\mu-1}\right) I_{k} & C \\
C^{T} & \left(\mu-s-r-\frac{s}{\mu-1}\right) I_{k}
\end{array}\right] .
\end{aligned}
$$

By Schur complement we have

$$
\begin{aligned}
& \operatorname{det}\left[\begin{array}{cc}
\left(\mu-m-r-\frac{m}{\mu-1}\right) I_{k} & C \\
C^{T} & \left(\mu-s-r-\frac{s}{\mu-1}\right) I_{k}
\end{array}\right] \\
= & \left(\mu-s-r-\frac{s}{\mu-1}\right)^{k} \operatorname{det}\left(\left(\mu-m-r-\frac{m}{\mu-1}\right) I_{k}-\frac{1}{\mu-s-r-\frac{s}{\mu-1}} C C^{T}\right) \\
= & \operatorname{det}\left(\left(\mu-m-r-\frac{m}{\mu-1}\right)\left(\mu-s-r-\frac{s}{\mu-1}\right) I_{k}-C C^{T}\right) \\
= & \left.f_{G}\left(\sqrt{\left(\mu-m-r-\frac{m}{\mu-1}\right)\left(\mu-s-r-\frac{s}{\mu-1}\right.}\right)\right) .
\end{aligned}
$$

So we obtain

$$
\begin{gathered}
f_{L\left(G_{m s}\right)}(\mu) \\
=\mu^{(m-s) k}(\mu-1)^{n s+(m-s) k} f_{G}\left(\sqrt{\left(\mu-m-r-\frac{m}{\mu-1}\right)\left(\mu-s-r-\frac{s}{\mu-1}\right)}\right)
\end{gathered}
$$

By the same argument of Theorem 2 we have the following theorem.

Theorem 3. Let $G$ be an $r$-regular bipartite graph with $|G|=n=2 k$ where $k$ is the size of each part. Then

$$
\begin{gathered}
f_{Q\left(G_{m s}\right)}(v) \\
\left.=v^{(m-s) k}(v-1)^{n s+(m-s) k} f_{G}\left(\sqrt{\left(v-m-r-\frac{m}{v-1}\right)\left(v-s-r-\frac{s}{v-1}\right.}\right)\right)
\end{gathered}
$$

Corollary 4. For graph $G^{\prime}$ we have

$$
\begin{aligned}
& f_{L\left(G^{\prime}\right)}(\mu)=(\mu-1)^{n s+(m-s) k} f_{G}\left(\sqrt{\left(\mu-m-r-\frac{m}{\mu-1}\right)\left(\mu-s-r-\frac{s}{\mu-1}\right)}\right) \\
& f_{Q\left(G^{\prime}\right)}(v)=(v-1)^{n s+(m-s) k} f_{G}\left(\sqrt{\left(v-m-r-\frac{m}{v-1}\right)\left(v-s-r-\frac{s}{v-1}\right)}\right) .
\end{aligned}
$$


Proof. The proof follows immediately from Theorems 2 and 3.

Corollary 5. For graph $G^{\prime}$, let $s=0$. Then

$$
\begin{gathered}
f_{L\left(G^{\prime}\right)}(\mu)=(\mu-1)^{m k} f_{G}\left(\sqrt{\left(\mu-m-r-\frac{m}{\mu-1}\right)(\mu-r)}\right), \\
f_{Q\left(G^{\prime}\right)}(v)=(v-1)^{m k} f_{G}\left(\sqrt{\left(v-m-r-\frac{m}{v-1}\right)(v-r)}\right) .
\end{gathered}
$$

Proof. Apply Corollary 4, for $s=0$.

Example 4. Let $G=K_{3,3}, m=3$ and $s=1$. Since $f_{G}(\lambda)=\lambda^{4}\left(\lambda^{2}-9\right)$ we have

$$
\begin{gathered}
f_{L\left(G^{\prime}\right)}(\mu)= \\
(\mu-1)^{12}\left(\mu-6-\frac{3}{\mu-1}\right)^{2}\left(\mu-4-\frac{1}{\mu-1}\right)^{2}\left(\left(\mu-6-\frac{3}{\mu-1}\right)\left(\mu-4-\frac{1}{\mu-1}\right)-9\right) \\
=(\mu-1)^{6}\left(\mu^{2}-7 \mu+3\right)^{2}\left(\mu^{2}-5 \mu+3\right)^{2}\left(\mu^{4}-12 \mu^{3}+8 \mu^{2}-18 \mu\right) \\
=\mu(\mu-1)^{6}\left(\mu^{2}-7 \mu+3\right)^{2}\left(\mu^{2}-5 \mu+3\right)^{2}\left(\mu^{3}-12 \mu^{2}+8 \mu-18\right) .
\end{gathered}
$$

So the Laplacian and signless Laplacian eigenvalues of the graph $G^{\prime}$ are

$$
\begin{gathered}
\left\{(0)^{[1]},(1)^{[6]},\left(\frac{7+\sqrt{37}}{2}\right)^{[2]},\left(\frac{7-\sqrt{37}}{2}\right)^{[2]},\left(\frac{5+\sqrt{13}}{2}\right)^{[2]},\left(\frac{5-\sqrt{13}}{2}\right)^{[2]}\right. \\
\left.,(8.47467)^{[1]},(2.75414)^{[1]},(0.77119)^{[1]}\right\} .
\end{gathered}
$$

The last three roots are found by newgraph.

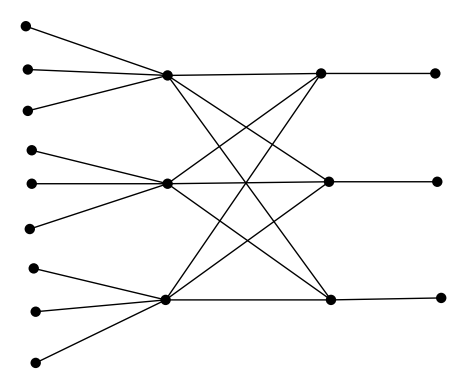

\section{REFERENCES}

[1] R. B. Bapat, Graphs and matrices. Springer, 2010.

[2] A. E. Brouwer and W. H. Haemers, Spectra of graphs. Springer Science \& Business Media, 2011.

[3] P. Csikvari, "Integral trees of arbitrarily large diameters," Journal of Algebraic Combinatorics, vol. 32, pp. 371-377, 2010, doi: 10.1007/s10801-010-0218-8.

[4] D. Cvetković, M. Doob, and H. Sachs, Spectra of Graph-Theory and Applications. Academic Press, New York, 1980. 
[5] D. Cvetković, P. Rowlinson, and S. Simić, An introduction to the theory of graph spectra, 2010.

[6] U. Knauer, Algebraic Graph Theory, ser. Morphisms, monoids and matrices. The Deutsche Nationalbibliothek, 2011.

Authors' addresses

A. R. Fiuj Laali

Department of Mathematics and Computer Science, Shahed University,Tehran, Iran.

E-mail address: a.fiujlaalieshahed.ac.ir

H. Haj Seyyed Javadi

Department of Mathematics and Computer Science, Shahed University,Tehran, Iran.

E-mail address: h.s.javadieshahed.ac.ir 